\title{
Decisive Role of Dark Matter in Cosmology
}

\author{
Vladimir S. Netchitailo \\ Biolase Inc., Irvine, CA, USA \\ Email: netchitailov@gmail.com
}

How to cite this paper: Netchitailo, V.S. (2022) Decisive Role of Dark Matter in Cosmology. Journal of High Energy Physics, Gravitation and Cosmology, 8, 115-142. https://doi.org/10.4236/jhepgc.2022.81009

Received: October 29, 2021

Accepted: December 28, 2021

Published: December 31, 2021

Copyright $\odot 2022$ by author(s) and Scientific Research Publishing Inc. This work is licensed under the Creative Commons Attribution International License (CC BY 4.0).

http://creativecommons.org/licenses/by/4.0/

\begin{abstract}
Hypersphere World-Universe Model (WUM) is an alternative to the prevailing Big Bang Model (BBM). WUM and BBM are principally different Models: 1) Instead of the Initial Singularity with the infinite energy density and the extremely rapid expansion of the space (Inflation) in BBM; in WUM, there was a Fluctuation (4D Nucleus of the World with an extrapolated radius equals to a basic unit of size a) in the Eternal Universe with a finite extrapolated energy density (four orders of magnitude less than the nuclear density) and a finite expansion of the Nucleus in its fourth spatial dimension with speed $c$ that is the gravitodynamic constant; 2) Instead of the Infinite Homogeneous and Isotropic Universe around the Initial Singularity in BBM; in WUM, the 3D Finite Boundless World (the Hypersphere of the 4D Nucleus) presents a Patchwork Quilt of different Luminous Superclusters $\left(\gtrsim 10^{3}\right)$, which emerged in various places of the World at different Cosmological times. The Medium of the World is Homogeneous and Isotropic. The distribution of Macroobjects in the World is spatially Inhomogeneous and Anisotropic and temporally Non-simultaneous. The Absolute Age of the entire World (determined by the parameters of the Medium) is $14.22 \mathrm{Gyr}$.
\end{abstract}

\section{Keywords}

Hypersphere World-Universe Model, Law of Conservation of Angular Momentum, Dark Epoch, Volcanic Rotational Fission, Luminous Epoch, Dark Matter Particles, Macroobject Shell Model, Medium of the World

\section{Introduction}

Hypersphere World-Universe Model (WUM) is an alternative to the prevailing Big Bang Model (BBM). They are principally different Models. Comparison of their main parameters is presented in Table 1.

WUM solves a number of physical problems in contemporary Cosmology through Dark Matter Particles (DMPs) and their interactions: Fermi Bubbles- 
two large structures in gamma-rays and X-rays above and below Galactic center; Coronal Heating problem in solar physics-temperature of Sun's corona exceeding that of photosphere by millions of degrees; Cores of Sun and Earth rotating faster than their surfaces; Diversity of Gravitationally-Rounded Objects in Solar system and their Internal Heating. WUM reveals Inter-Connectivity of Primary Cosmological Parameters and calculates their values, which are in good agreement with the latest results of their measurements.

Table 1. Parameters of big bang model and world-universe model [16].

\begin{tabular}{|c|c|c|}
\hline Parameter & Big Bang Model & World-Universe Model \\
\hline $\begin{array}{l}\text { Structure of } \\
\text { the World }\end{array}$ & $3+1$ Spacetime & $\begin{array}{l}3 \mathrm{D} \text { Hypersphere of } 4 \mathrm{D} \text { Nucleus of } \\
\text { the World. } \\
\text { Time is a Factor of the World }\end{array}$ \\
\hline The Beginning & Initial Singularity & $\begin{array}{l}4 \mathrm{D} \text { Nucleus of the World with an } \\
\text { extrapolated radius a as the result of } \\
\text { a fluctuation in the Eternal Universe }\end{array}$ \\
\hline Expansion & $\begin{array}{l}\text { Inflation-extremely } \\
\text { rapid expansion } \\
\text { of space }\end{array}$ & $\begin{array}{l}\text { Radius of the } 4 \mathrm{D} \text { Nucleus of the World } \\
\text { is increasing with speed } c \text { that is the } \\
\text { gravitodynamic constant }\end{array}$ \\
\hline Cosmological & Homogeneous and & $\begin{array}{l}\text { Homogeneous and Isotropic Medium } \\
\text { of the World }\end{array}$ \\
\hline Principal & Isotropic Universe & $\begin{array}{l}\text { Inhomogeneous and Anisotropic } \\
\text { distribution of Macroobjects }\end{array}$ \\
\hline Content & $\begin{array}{l}\text { Dark Energy, Cold } \\
\text { Dark Matter, } \\
\text { Ordinary Matter }\end{array}$ & $\begin{array}{l}\text { Multicomponent Dark Matter (DM), } \\
\text { Ordinary Matter }\end{array}$ \\
\hline Origin of Matter & Initial Singularity & $\begin{array}{l}\text { DM comes from the Universe to the } \\
\text { Nucleus along Its fourth spatial } \\
\text { dimension. Ordinary Matter is } \\
\text { a byproduct of DMPs self-annihilation }\end{array}$ \\
\hline $\begin{array}{l}\text { Cosmic } \\
\text { Microwave } \\
\text { Background }\end{array}$ & $\begin{array}{l}\text { Photon's wavelength } \\
\text { is increasing over time }\end{array}$ & $\begin{array}{l}\text { Thermodynamic equilibrium of } \\
\text { photons with Intergalactic plasma }\end{array}$ \\
\hline $\begin{array}{l}\text { Nucleosynthesis } \\
\text { of Light Elements }\end{array}$ & $\begin{array}{l}\text { Big Bang } \\
\text { Nucleosynthesis }\end{array}$ & $\begin{array}{l}\text { Nucleosynthesis of all elements } \\
\text { (including light } \\
\text { elements) occurs inside of DM Cores } \\
\text { of Macroobjects }\end{array}$ \\
\hline $\begin{array}{l}\text { Primary } \\
\text { Cosmological } \\
\text { Parameters }\end{array}$ & Independent & Inter-connected \\
\hline Galactic Centre & Black Hole & DM Core of Galaxy \\
\hline $\begin{array}{l}\text { Law of } \\
\text { Conservation } \\
\text { of Angular } \\
\text { Momentum }\end{array}$ & Inconsistent & Consistent \\
\hline
\end{tabular}


In 2013, WUM predicted the values of the following Cosmological parameters: Hubble's, Gravitational, Intergalactic plasma concentration, and Photons minimum energy, which were experimentally confirmed in 2015-2021. "The Discovery of a Supermassive Compact Object at the Centre of Our Galaxy" (Nobel Prize in Physics 2020) made by Prof. R. Genzel and A. Ghez is a confirmation of one of the most important predictions of WUM in 2013: "Macroobjects of the World have cores made up of the discussed DM particles. Other particles, including DM and baryonic matter, form shells surrounding the cores" [1].

This manuscript concludes the series of papers on WUM published by "Journal of High Energy Physics, Gravitation and Cosmology" journal [2]-[21]. Many results obtained there are quoted in the current work without a full justification; an interested reader is encouraged to view the referenced papers in such cases.

\section{History of Dark Matter}

\subsection{Early Ideas}

The history of DM can be traced back to at least the middle of the 19th century.

G. Bertone and D. Hooper provide an excellent review of this history [22]:

- In 1844, F. Bessel argued that the observed proper motion of the stars Sirius and Procyon could only be explained by the presence of faint companion stars influencing the observed stars through their gravitational pull: If we were to regard Procyon and Sirius as double stars, their change of motion would not surprise us. The existence of numberless visible stars can prove nothing against the evidence of numberless invisible ones,

- In 1846, U. Le Verrier and J. C. Adams, in order to explain some persistent anomalies in the motion of Uranus, proposed the existence of a new planet;

- Beside dark stars and planets, astronomers in the 19th century also discussed dark matter in the form of dark clouds, or dark "nebulae". In 1877, A. Secchi wrote: Among these studies there is the interesting probable discovery of dark masses scattered in space, whose existence was revealed thanks to the bright background on which they are projected. Until now they were classified as black cavities, but this explanation is highly improbable, especially after the discovery of the gaseous nature of the nebular masses,

- As soon as astronomical photography was invented, scientists started to notice that stars were not distributed evenly on the sky. Dark regions were observed in dense stellar fields. In 1894, A. Ranyard wrote: The dark vacant areas or channels running north and south, in the neighborhood of [ $\theta$ Ophiuchi] at the center ... seem to me to be undoubtedly dark structures, or absorbing masses in space, which cut out the light from the nebulous or stellar region behind them;

- In 1904, Lord Kelvin was among the first to attempt a dynamical estimate of the amount of dark matter in the Milky Way. His argument was simple yet powerful: if stars in the Milky Way can be described as a gas of particles, act- 
ing under the influence of gravity, then one can establish a relationship between the size of the system and the velocity dispersion of the stars: It is nevertheless probable that there may be as many as $10^{9}$ stars (within a sphere of radius $3.09 \times 10^{16} \mathrm{~km}$ ) but many of them may be extinct and 10 dark, and nine-tenths of them though not all dark may be not bright enough to be seen by us at their actual distances. [...] Many of our stars, perhaps a great majority of them, may be dark bodies;

- H. Poincare was impressed by Lord Kelvin's idea of applying the "theory of gases" to the stellar system of Milky Way. In 1906, he explicitly mentioned "dark matter" and argued that since the velocity dispersion predicted in Kelvin's estimate is of the same order of magnitude as that observed, the amount of dark matter was likely to be less than or similar to that of visible matter;

- J. Kapteyn was among the first to offer a quantitative model for the shape and size of the Galaxy, describing it as a flattened distribution of stars, rotating around an axis that points towards the Galactic Pole. He argued that the Sun was located close to the center of the Galaxy, and that the motion of stars could be described as that of a gas in a quiescent atmosphere. In 1922, he explicitly addressed the possible existence of dark matter in the Galaxy: We therefore have the means of estimating the mass of the dark matter in the universe. As matters stand at present, it appears at once that this mass cannot be excessive. If it were otherwise, the average mass as derived from binary stars would have been very much lower than what has been found for the effective mass,

- In 1932, Kapteyn's pupil J. Oort derived a most probable value for the total density of matter near the Sun of $6.3 \times 10^{-24} \mathrm{~g} \cdot \mathrm{cm}^{-3}$. It is interesting to recall the words used by Oort to illustrate the constraint on the amount of dark matter: We may conclude that the total mass of nebulous or meteoric matter near the sun is less than $3 \times 10^{-24} \mathrm{~g} \cdot \mathrm{cm}^{-3}$; it is probably less than the total mass of visible stars, possibly much less;

- In 1930, K. Lundmark measured the galaxy rotation curves of several different galaxies and compared the mass required to the luminous mass of the galaxies. His conclusion was the same as that of V. Rubin 40 years later, a large part of the mass of a galaxy is in the form which is not visible to us. Like Zwicky would do three years later, Lundmark spoke about this additional mass as "Dunkle Materie" or, literally translated, "Dark Matter" [23];

- In 1933, F. Zwicky investigated the velocity dispersion of the Coma cluster and found a surprisingly high mass-to-light ratio ( 500$)$. He concluded: if this would be confirmed, we would get the surprising result that dark matter is present in much greater amount than luminous matter,

- What did Zwicky think that the dark matter in Coma and other galaxy clusters might be? An illuminating sentence in his 1937 paper provides a rather clear answer to this question: In order to derive the mass of galaxies from their luminosity we must know how much dark matter is incorporated in nebulae in the form of cool and cold stars, macroscopic and microscopic sol- 
id bodies, and gases;

- From our contemporary perspective, it can be easy to imagine that F. Zwicky, V. Rubin, and the other early dark matter pioneers had halos of weakly interacting particles in mind when they discussed dark matter. In reality, however, they did not. But over time an increasing number of particle physicists became interested in cosmology, and eventually in the problem of dark matter.

\subsection{Recent Developments}

Our article "Astrophysics: Macroobject Shell Model" focuses on more recent developments [9]:

- The prospect that Dark Matter Particles (DMPs) might be observed in Centers of Macroobjects has drawn many new researchers to the field in the last forty-four years. In 1977-1980, indirect effects in cosmic rays and gamma-ray background from the annihilation of Cold DM in the form of heavy stable neutral leptons in Galaxies were considered in pioneer articles [24]-[29];

- In the wake of the failures of hot DM, it was quickly becoming appreciated that cold DM could do a much better job of accounting for the observed patterns of large-scale structure. In 1984, G. Blumenthal, S. Faber, J. Primack, and M. Rees wrote: "We have shown that a universe with $\sim 10$ times as much cold dark matter as baryonic matter provides a remarkably good fit to the observed universe. This model predicts roughly the observed mass range of galaxies, the dissipational nature of galaxy collapse, and the observed Faber-Jackson and Tully-Fisher relations. It also gives dissipationless galactic halos and clusters. In addition, it may also provide natural explanations for galaxy-environment correlations and for the differences in angular momenta between ellipticals and spiral galaxies" [22];

- Although the term WIMPs (weakly interacting massive particles), as coined by G. Steigman and M. Turner in 1984, was originally intended to include all particle dark matter candidates, including axions, gravitinos, etc., the definition of this term has since evolved to denote only more often those particles that interact through the weak force [22];

- By the end of the 1980s, the conclusion that most of the mass in the Universe consists of cold and non-baryonic particles had become widely accepted, among many astrophysicists and particle physicists alike. Cold dark matter in the form of some unknown species of elementary particle had become the leading paradigm [22];

- The role of cold DM in the formation of Primordial Luminous Objects is discussed by E. Ripamonti and T. Abel [30];

- A mechanism whereby DM in protostellar halos plays a role in the formation of the first stars is discussed by D. Spolyar, K. Freese and P. Gondolo [31]. Heat from neutralino DM annihilation is shown to overwhelm any cooling mechanism, consequently impeding the star formation process. A "dark star" powered by DM annihilation instead of nuclear fusion may result [31]. Dark 
stars are in hydrostatic and thermal equilibrium, but with an unusual power source. Weakly Interacting Massive Particles (WIMPs) are among the best candidates for DM [32];

- Important cosmological problems like Dark Matter and Dark Energy could be, in principle, solved through extended gravity that is stressed by C. Corda [33].

- Two-component DM systems consisting of bosonic and fermionic components are proposed for the explanation of emission lines from the bulge of the Milky Way galaxy. C. Boehm, P. Fayet, J. Silk analyze the possibility of two coannihilating neutral and stable DMPs: a heavy fermion for example, like the lightest neutralino $(>100 \mathrm{GeV})$ and the other one a possibly light spin-0 particle $(\sim 100 \mathrm{MeV})[34]$;

- Conversions and semi-annihilations of DMPs in addition to the standard DM annihilations are considered in a three-component DM system [35]. Multicomponent DM models consisting of both bosonic and fermionic components were analyzed in literature (for example, see [36] [37] [38] [39] [40] and references therein).

\section{Dark Matter in WUM}

\subsection{Multicomponent Dark Matter}

WUM proposes multicomponent DM system consisting of two couples of coannihilating DMPs: a heavy Dark Matter Fermion (DMF)_DMF1 (1.3 TeV) and a light spin-0 boson-DIRAC (70 MeV) that is a dipole of Dirac's monopoles with charge $\mu=e / 2 \alpha$ ( $e$ is the elementary charge); a heavy fermion-DMF2 (9.6 $\mathrm{GeV})$ and a light spin-0 boson-ELOP $(340 \mathrm{keV})$ that is a dipole of preons with electrical charge $e / 3$; a self-annihilating fermion-DMF3 (3.7 keV), and a fermion DMF4 $(0.2 \mathrm{eV})$.

WUM postulates that rest energies of DMFs and bosons are proportional to a basic unit of energy $E_{0}=h c / a$ multiplied by different exponents of $\alpha$ and can be expressed with the following formulae [4]:

$$
\begin{aligned}
& \text { DMF1 (fermion): } \quad E_{D M F 1}=\alpha^{-2} E_{0}=1.3149950 \mathrm{TeV} \\
& \text { DMF2 (fermion): } \quad E_{D M F 2}=\alpha^{-1} E_{0}=9.5959823 \mathrm{GeV} \\
& \text { DIRAC (boson): } \quad E_{D I R A C}=\alpha^{0} E_{0}=70.025267 \mathrm{MeV} \\
& \text { ELOP (boson): } \quad E_{E L O P}=2 / 3 \alpha^{1} E_{0}=340.66606 \mathrm{keV} \\
& \text { DMF3 (fermion): } \quad E_{D M F 3}=\alpha^{2} E_{0}=3.7289402 \mathrm{keV} \\
& \text { DMF4 (fermion): } \quad E_{D M F 4}=\alpha^{4} E_{0}=0.19857111 \mathrm{eV}
\end{aligned}
$$

where $h$ is Planck constant; $\alpha$ is the dimensionless Rydberg constant: $\alpha=\left(2 a R_{\infty}\right)^{1 / 3}$ (that was later named "Fine-structure constant"); $a$ is a basic unit of size $a=1.7705641 \times 10^{-14} \mathrm{~m}$; and $c$ is the gravitodynamic constant that is the ratio of the absolute gravitomagnetic unit of charge $E_{0}$ to the absolute gravitostatic 
unit of charge $E_{0} / c$. It is worth noting that the speed of light in vacuum, commonly denoted as $c$, is not related to the World in our Model, because there is no vacuum in it. Instead, there is the Medium of the World consisting of elementary particles. Also note that the rest energy of electron $E_{e}$ equals to: $E_{e}=\alpha E_{0}$ and the Rydberg unit of energy is: $R y=h c R_{\infty}=0.5 \alpha^{3} E_{0}=13.605693 \mathrm{eV}$.

We still do not have a direct confirmation of DMPs' rest energies, but we do have a number of indirect observations. The signatures of DMPs self-annihilation with expected rest energies of $1.3 \mathrm{TeV}$; $9.6 \mathrm{GeV}$; $70 \mathrm{MeV}$; $340 \mathrm{keV}$; $3.7 \mathrm{keV}$ are found in spectra of the diffuse gamma-ray background and the emissions of various Macroobjects in the World. We connect observed gamma-ray spectra with the structure of Macroobjects (nuclei and shells composition). Self-annihilation of those DMPs can give rise to any combination of gamma-ray lines. Thus, the diversity of Very High Energy gamma-ray sources in the World has a clear explanation in WUM [9].

In this regard, it is worth recalling a story about neutrinos: "The neutrino was postulated first by W. Pauli in 1930 to explain how beta decay could conserve energy, momentum, and angular momentum (spin). But we still don't know the values of neutrino masses". Although we still cannot measure neutrinos' masses directly, no one doubts their existence [4].

\subsection{Weak Interaction}

The widely discussed models for nonbaryonic DM are based on the Cold DM hypothesis, and corresponding particles are commonly assumed to be WIMPs, which interact via gravity and any other force (or forces), potentially not part of the standard model itself, which is as weak as or weaker than the weak nuclear force, but also, non-vanishing in its strength [Wikipedia. Weakly interacting massive particles]. It follows that a new weak force needs to exist, providing interaction between DMPs. The strength of this force exceeds that of gravity, and its range is considerably greater than that of the weak nuclear force.

According to WUM, strength of gravity is characterized by gravitational parameter $G$ [17]:

$$
G=G_{0} \times Q^{-1}
$$

where $G_{0}=\frac{a^{2} c^{4}}{8 \pi h c}$ is an extrapolated value of $G$ at the Beginning of the World $(Q=1)$. A dimensionless time-varying quantity $Q$, which is a measure of the Size $R$ and Age $A_{c}$ of the World and is, in fact, the "Dirac Large Number" $\left(t_{0}\right.$ is a basic unit of time: $t_{0}=a / c=5.9059662 \times 10^{-23} \mathrm{~s}$ ):

$$
Q=\frac{R}{a}=\frac{A_{c}}{t_{0}}
$$

in present epoch equals to: $Q=0.759972 \times 10^{40}$. The range of the gravity equals to the size of the World $R$ : 


$$
R=a \times Q=1.34558 \times 10^{26} \mathrm{~m}
$$

In WUM, a weak interaction is characterized by the parameter $G_{W}$ :

$$
G_{W}=G_{0} \times Q^{-1 / 4}
$$

which is about thirty orders of magnitude greater than $G$. The range of the weak interaction $R_{W}$ in the present Epoch equals to:

$$
R_{W}=a \times Q^{1 / 4}=1.65314 \times 10^{-4} \mathrm{~m}
$$

that is much greater than the range of the weak nuclear force. Calculated concentration of DMF4 particles $n_{D M F}$ in the largest shell of Superclusters: $n_{D M F} \cong 4.2 \times 10^{15} \mathrm{~m}^{-3}$ (see Table 2 ) shows that a distance between particles is around $\sim 10^{-5} \mathrm{~m}$, which is much smaller than $R_{W}$. Thus, the introduced weak interaction between DMPs will provide integrity of all DM shells. In our view, weak interaction between particles DMF3 provides integrity of Fermi Bubbles (see Section 4.7.).

\subsection{Macroobject Shell Model}

In WUM, Macrostructures of the World (Superclusters, Galaxies, Extrasolar systems) have Nuclei made up of DMFs, which are surrounded by Shells composed of DM and Baryonic Matter. The shells envelope one another, like a Russian doll. The lighter a particle, the greater the radius and the mass of its shell. Innermost shells are the smallest and are made up of heaviest particles; outer shells are larger and consist of lighter particles. Introduced principally new Weak Interaction of DMPs with Matter provides integrity of all shells: a distance between particles is smaller than the range of the weak interaction (see Section 3.2). Table 2 describes the parameters of Macroobjects' Cores, which are 3D fluid balls with a high viscosity and act as solid-state objects, made up of different fermions.

The calculated parameters of the shells show that [8]:

- Nuclei made up of DMF1 and/or DMF2 compose Cores of stars in Extrasolar Systems;

Table 2. Parameters of Macroobjects' Cores made up of different Fermions in present Epoch.

\begin{tabular}{ccccc}
\hline Fermion & $\begin{array}{c}\text { Fermion Mass } \\
\boldsymbol{m}_{\mathbf{f}, ~} \mathbf{M e V}\end{array}$ & $\begin{array}{c}\text { Macroobject } \\
\text { Mass } \boldsymbol{M}_{\max }, \\
\mathbf{~ k g}\end{array}$ & $\begin{array}{c}\text { Macroobject } \\
\text { Radius } \\
\boldsymbol{R}_{\min }, \mathbf{m}\end{array}$ & $\begin{array}{c}\text { Macroobject } \\
\text { Density } \boldsymbol{\rho}_{\max }, \\
\mathbf{k g} \cdot \mathbf{m}^{-3}\end{array}$ \\
\hline DMF1 & $1.3 \times 10^{6}$ & $1.9 \times 10^{30}$ & $8.6 \times 10^{3}$ & $7.2 \times 10^{17}$ \\
DMF2 & $9.6 \times 10^{3}$ & $1.9 \times 10^{30}$ & $8.6 \times 10^{3}$ & $7.2 \times 10^{17}$ \\
Electron-Positron & 0.51 & $6.6 \times 10^{36}$ & $2.9 \times 10^{10}$ & $6.3 \times 10^{4}$ \\
DMF3 & $3.7 \times 10^{-3}$ & $1.2 \times 10^{41}$ & $5.4 \times 10^{14}$ & $1.8 \times 10^{-4}$ \\
DMF4 & $2 \times 10^{-7}$ & $4.2 \times 10^{49}$ & $1.9 \times 10^{23}$ & $1.5 \times 10^{-21}$ \\
\hline
\end{tabular}


- Shells of DMF3 and/or Electron-Positron plasma around Nuclei made up of DMF1 and/or DMF2 make up Cores of Galaxies;

- Nuclei made up of DMF1 and/or DMF2 surrounded by shells of DMF3 and DMF4 compose Cores of Superclusters.

In our view, Macroobjects of the World possess the following properties [9]:

- Nuclei are made up of DMPs. Surrounding shells contain DM and Baryonic matter;

- Nuclei and shells are growing in time proportionally to square root of cosmological time $\propto \tau^{1 / 2}$ until one of them reaches a critical point of its local stability, at which it detonates. The energy released during detonation is produced by the self-annihilation of DMPs. The detonation process does not destroy the Macroobject; instead, Hyper-flares occur in active areas of shells, analogous to Solar flares;

- All other DMPs in different shells can start self-annihilation process as the result of the first detonation;

- Different emission lines in spectra of bursts are connected to the Macroobjects' structure which depends on the composition of Nuclei and surrounding shells made up of DMPs. Consequently, the diversity of Very High Energy Bursts has a clear explanation;

- Afterglow is a result of processes developing in Nuclei and shells after detonation.

\subsection{Macrostructures}

Laniakea Supercluster (LSC) is a galaxy supercluster that is home to Milky Way (MW) and approximately 100,000 other nearby galaxies (see Figure 1). It is

Our cosmic address

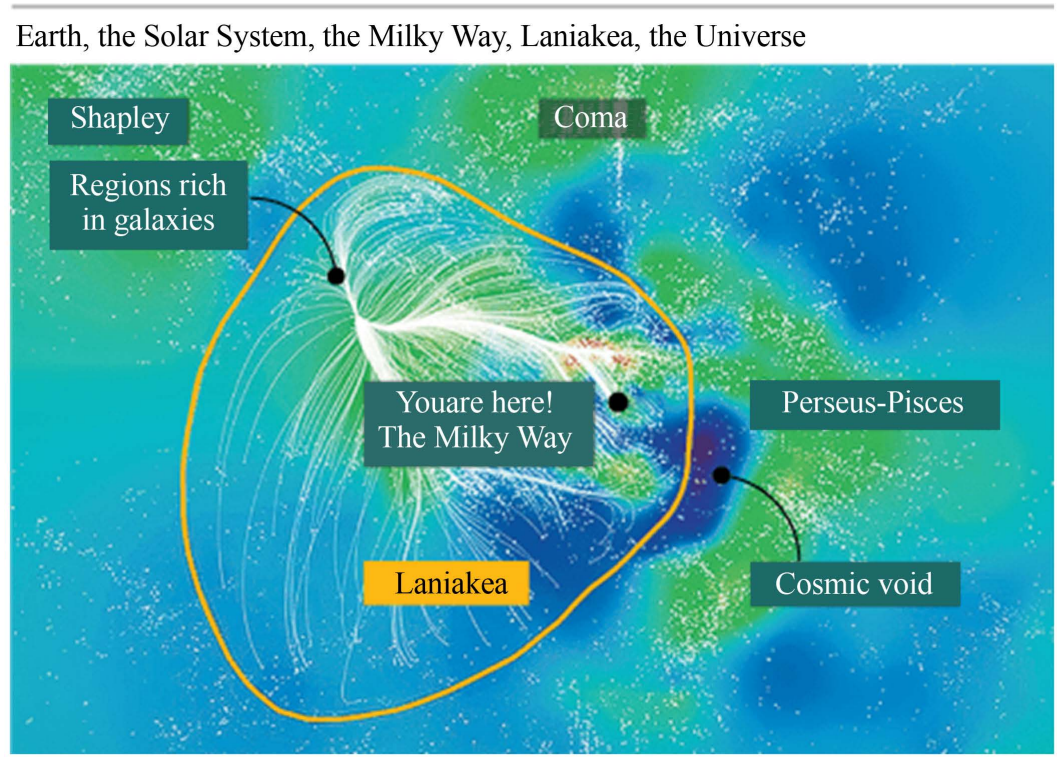

Figure 1. Laniakea supercluster. Adapted from [44]. 
known as one of the largest superclusters with estimated binding mass $10^{17} M_{\odot}$ [41]. The neighboring superclusters to LSC are the Shapley Supercluster, Hercules Supercluster, Coma Supercluster, and Perseus-Pisces Supercluster. Distance from the Earth to the Centre of LSC is $250 \mathrm{Mly}$. The mass-to-light ratio of the Virgo Supercluster is about three hundred times larger than that of the Solar ratio. Similar ratios are obtained for other superclusters [42]. In 1933, F. Zwicky investigated the velocity dispersion of Coma cluster and found a surprisingly high mass-to-light ratio ( 500). He concluded: "If this would be confirmed, we would get the surprising result that dark matter is present in much greater amount than luminous matter" [43]. These ratios are one of the main arguments in favor of presence of significant amounts of DM in the World.

We emphasize that about 100,000 nearby galaxies are moving around Centre of Laniakea Supercluster. They belong to LSC. All these galaxies did not start their movement from the "Initial Singularity". The neighboring superclusters have the same structure (see Figure 2). It means that the World is, in fact, a Patchwork Quilt of different Luminous Superclusters $\left(\gtrsim 10^{3}\right)$ [21].

According to R. B. Tully, et al., "Galaxies congregate in clusters and along filaments, and are missing from large regions referred to as voids. These structures are seen in maps derived from spectroscopic surveys that reveal networks of structure that are interconnected with no clear boundaries. Extended regions with a high concentration of galaxies are called 'superclusters', although this term is not precise" [44].

P. Wang, et al. made a great discovery: "Most cosmological structures in the universe spin. Although structures in the universe form on a wide variety of scales

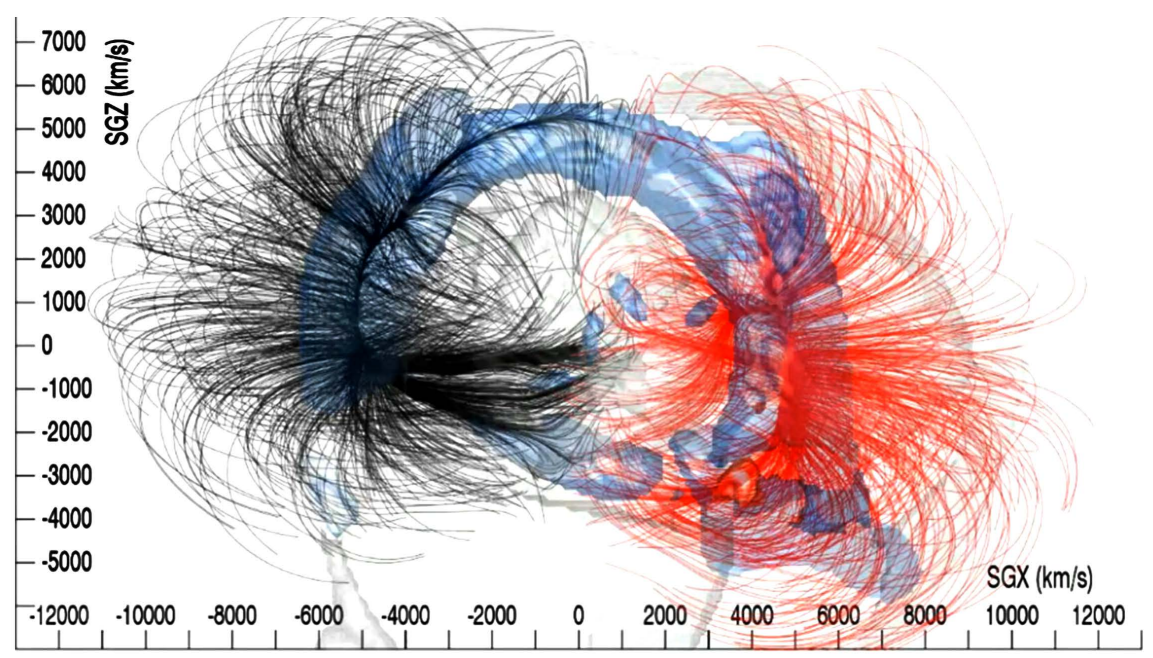

Figure 2. A representation of structure and flows due to mass within $6000 \mathrm{~km} \cdot \mathrm{s}^{-1}(\sim 80$ $\mathrm{Mpc}$ ). Surfaces of red and blue respectively represent outer contours of clusters and filaments as defined by the local eigenvalues of the velocity shear tensor determined from the Wiener Filter analysis. Flow threads originating in our basin of attraction that terminate near the Norma Cluster are in black and adjacent flow threads that terminate at the relative attractor near the Perseus Cluster are in red. The Arch and extended Antlia Wall structures bridge between the two attraction basins. Adapted from [44]. 
from small dwarf galaxies to large super clusters, the generation of angular momentum across these scales is poorly understood. We have investigated the possibility that filaments of galaxies-cylindrical tendrils of matter hundreds of millions of light-years across, are themselves spinning. By stacking thousands of filaments together and examining the velocity of galaxies perpendicular to the filament's axis (via their red and blue shift), we have found that these objects too display motion consistent with rotation making them the largest objects known to have angular momentum. These results signify that angular momentum can be generated on unprecedented scales" [45].

In June 2021, at the "Giant Arc at the 238th virtual meeting of the American Astronomical Society", A. Lopez reported about the discovery of "a giant, almost symmetrical arc of galaxies- the Giant Arc-spanning 3.3 billion light years at a distance of more than 9.2 billion light years away that is difficult to explain in current models of the Universe. The Giant Arc, which is approximately 1/15th the radius of the observable universe, is twice the size of the striking Sloan Great Wall of galaxies and clusters that is seen in the nearby Universe. This new discovery of the Giant Arc adds to an accumulating set of (cautious) challenges to the Cosmological Principle. The discovery of the Giant Arc adds to the number of structures on scales larger than those thought to be "smooth," and therefore pushes the boundary size for the Cosmological Principle. The growing number of large-scale structures over the size limit of what is considered theoretically viable is becoming harder to ignore. According to cosmologists, the current theoretical limit is calculated to be 1.2 billion light years, which makes the Giant Arc almost three times larger. Can the standard model of cosmology account for these huge structures in the Universe as just rare flukes or is there more to it than that?' [46].

B. Carr, et al. "consider the observational constraints on stupendously large black holes (SLABS) in the mass range $M>10^{11} M_{\odot}$. These have attracted little attention hitherto, and we are aware of no published constraints on a SLAB population in the range $\left(10^{12}-10^{18}\right) M_{\odot}$. However, there is already evidence for black holes of up to nearly $10^{11} \mathrm{M}_{\odot}$ in galactic nuclei, so it is conceivable that SLABs exist, and they may even have been seeded by primordial black holes" [47].

WUM. These latest observations of the World can be explained in frames of the developed WUM only:

- "Galaxies do not congregate in clusters and along filaments." On the contrary, Cosmic Web that is "networks of structure that are interconnected with no clear boundaries" is the result of the Rotational Fission of DM Cores of neighbor Superclusters;

- "Generation of angular momentum across these scales" provide DM Cores of Superclusters through the Rotational Fission mechanism;

- "Spinning cylindrical tendrils of matter hundreds of millions of light-years across" are the result of spiral jets of galaxies generated by DM Cores of Superclusters with internal rotation; 
- The Giant Arc is the result of the intersection of the Galaxies' jets generated by the neighbor DM Cores of Superclusters;

- The calculated maximum mass of the supercluster DM Core of $2.1 \times 10^{19}$ solar mass (see Table 2) is in good agreement with the values discussed by $\mathrm{L}$. Bliss [41] and B. Carr, F. Kühnel and L. Visinelli [47]. In the future, these stupendously large compact objects can give rise to new Luminous Superclusters as the result of their DM Cores' rotational fission;

- 13.77 Gyr ago, when the Laniakea Supercluster emerged, the estimated number of DM Supercluster Cores in the World was around $\sim 10^{3}$ [21]. It is unlikely that all of them gave birth to Luminous Superclusters at the same cosmological time being far away from each other. The 3D Finite Boundless World presents a Patchwork Quilt of different Luminous Superclusters, which emerged at different Cosmological times.

\section{Dark Matter Cosmology}

\subsection{Medium of the World}

WUM introduces the Medium of the World, which consists of stable elementary particles with lifetimes longer than the age of the World: protons, electrons, photons, neutrinos, and Dark Matter Particles (DMPs). The existence of the Medium is a principal point of WUM. It follows from the observations of Intergalactic Plasma; Cosmic Microwave Background Radiation (MBR); Far-Infrared Background Radiation. Inter-galactic voids discussed by astronomers are, in fact, examples of the Medium in its purest. MBR is part of the Medium; it then follows that the Medium is the absolute frame of reference. Relative to the MBR rest frame, the Milky Way galaxy and the Sun are moving with the speed of 552 and $370 \mathrm{~km} \cdot \mathrm{s}^{-1}$, respectively [6].

The energy density of the Medium is $2 / 3$ of the total energy density of the World. Superclusters, Galaxies, Extrasolar systems, planets, moons are made of the same particles. The energy density of Macroobjects adds up to $1 / 3$ of the total energy density of the World throughout the World's evolution [6].

Cosmological principal is valid for the Homogeneous and Isotropic Medium. The distribution of Macroobjects is Inhomogeneous and Anisotropic, and therefore, the Cosmological Principal is not viable for the entire World.

WUM is the classical model, therefore classical notions can be introduced only when the very first ensemble of particles was created at the cosmological time $\tau_{M}$ equals to: $\tau_{M}=\alpha^{-2} \times t_{0} \cong 10^{-18} \mathrm{~S} \quad$ [14]. The cosmological principal Universality of Physical Laws is valid at the cosmological times $\tau \geq \tau_{M}$ because Physical Laws are determined by the Medium of the World.

In frames of WUM, Time and Space are closely connected with the Mediums' impedance (wave resistance) $Z_{g}$ that equals to the Hubble's parameter $H$ : $Z_{g}=H=\tau^{-1}$ and the gravitomagnetic parameter $\mu_{g}$, which equals to: $\mu_{g}=R^{-1}$. It follows that neither Time nor Space could be discussed in absence of the Medium. 
According to WUM, the World is the 3D Hypersphere of the 4D Nucleus, which is expanding in Its fourth spatial dimension. All points of the Hypersphere are equivalent; there are no preferred centers or boundaries of the World. A Hypersphere is an example of a 3-Manifold which locally behaves like regular Euclidean 3D space: just as a sphere looks like a plane to small enough observers. The 3D Finite Boundless World has a Spatial Measure - Radius of the curvature of the $4 \mathrm{D}$ Nucleus $R$. All spatial parameters of the World can be measured relatively to $R$. Any cosmological model of the Infinite Universe has no Spatial Measure.

WUM introduces a Cosmological Time that is principally different from the Solar Time, which is defined by the parameters of the Solar System, and Cosmic Time of the General Relativity. It is defined by the Impedance of the Medium of the World that equals to the Hubble's parameter. Cosmological Time $\tau$ marches on at constant pace since the Beginning of the World until the present Epoch and is, in fact, a Timing Measure that defines the Age of the World $A_{\tau}=\tau$. All timing parameters of the World can be measured relatively to the Age of the World. WUM concludes that any theory of evolution of the World should be consistent with the Cosmological Time [18]. In our everyday life we use the alleged Space (3D Euclidean) and Solar Time.

The gravitational parameter $G$ that is proportional to the Mediums' energy density can be introduced only for the Medium filled with Matter. The Gravitation is a result of simple interactions of DMPs with Matter (by the introduced new Weak interaction) that work cooperatively to create a more complex interaction. DMPs are responsible for the Le Sage's mechanism of the gravitation [5]. Gravity, Space and Time are all emergent phenomena [14]. In this regard, it is worth recalling Albert Einstein quote: "When forced to summarize the theory of relativity in one sentence: time and space and gravitation have no separate existence from matter."

\subsection{Creation of Matter}

WUM follows the idea of the continuous creation of matter by the additive mechanism discussed by P. Dirac in 1974 [48]. To provide the creation of Matter by the Universe uniformly throughout the World, we consider the following Concept of the World proposed by G. Riemann in 1854 [49]: 3D Finite World is a Hypersphere of $4 \mathrm{D}$ Nucleus. In our view, the World was started by a Fluctuation in Eternal Universe, and 4D Nucleus of the World with a radius of a was born. The Nucleus is expanding in its fourth spatial dimension and Its surface, the Hypersphere, is likewise expanding. The radius of the Nucleus $R$ is increasing with the speed $c$ (gravitodynamic constant) for a cosmological time $\tau$ from the Beginning and equals $R=c \tau$.

The surface of the Nucleus is created in a process analogous to sublimation. Continuous creation of matter is the result of this process. Sublimation is a well-known endothermic process that happens when surfaces are intrinsically 
more energetically favorable than the bulk of a material, and hence there is a driving force for surfaces to be created. DM is created by the Universe in the 4D Nucleus of the World. DMPs carry new DM into the 3D Hypersphere World. Ordinary Matter is a byproduct of DMPs self-annihilation. Consequently, a Matter-Antimatter Asymmetry problem discussed in literature does not arise (since antimatter does not get created by DMPs self-annihilation). By analogy with 3D ball, which has 2D spherical surface (that has surface energy), we can imagine that the 3D Hypersphere World has a "Surface Energy" of the 4D Nucleus.

The proposed 4D process is responsible for the Expansion, Creation of Matter, and Arrow of Time. It constitutes the main Hypothesis of WUM. In our view, the arrow of the Cosmological Time does not depend on any physical phenomenon in the Medium of the World. It is the result of the Worlds' expansion due to the driving force for surfaces to be created [14]. It is important to emphasize that:

- Creation of Matter is a direct consequence of expansion;

- Creation of DM occurs homogeneously in all points of the 3D Finite Boundless Hypersphere World.

\subsection{Angular Momentum Problem}

Angular momentum problem is one of the most critical problems in Standard Cosmology that must be solved. Standard Cosmology does not explain how Galaxies and Extrasolar systems obtained their enormous orbital angular momenta [12]:

- Solar System (SS) has an orbital momentum $L_{\text {orb }}^{S S}$ calculated based on the distance of 26.4 kly from the galactic Centre and orbital speed of about 220 $\mathrm{km} / \mathrm{s}: \quad L_{\text {orb }}^{S S}=1.1 \times 10^{56} \mathrm{~J} \cdot \mathrm{s}$, which far exceeds the rotational angular momentum: $L_{\text {rot }}^{S S}=3.2 \times 10^{43} \mathrm{~J} \cdot \mathrm{s}$;

- Milky Way (MW) galaxy is gravitationally bounded with the Virgo Supercluster and has an orbital angular momentum $L_{\text {orb }}^{M W}$ calculated based on the distance of 65 million light-years from Virgo Supercluster and orbital speed of about $400 \mathrm{~km} / \mathrm{s}[50]: L_{\text {orb }}^{M W}=2.5 \times 10^{71} \mathrm{~J} \cdot \mathrm{s}$, which far exceeds the total rotational angular momentum of MW [12]: $L_{\text {rot }}^{M W} \approx 1 \times 10^{67} \mathrm{~J} \cdot \mathrm{s}$.

In our opinion, there is only one mechanism that can supply angular momenta to Macroobjects-Rotational Fission of overspinning (surface speed at equator exceeding escape velocity) Prime Objects. From the point of view of Fission model, the Prime Object is transferring some of its rotational angular momentum to orbital and rotational momenta of satellites. It follows that the rotational momentum of the prime object should exceed the orbital momentum of its satellite.

In frames of WUM, Prime Objects are DM Cores of Superclusters, which must accumulate tremendous rotational angular momenta before the Birth of the Luminous World. This process must take a long enough time in the history 
of the World, which we named "Dark Epoch" [12].

\subsection{Dark Epoch}

Dark Epoch started at the Beginning of the World and lasted for $0.45 \mathrm{Gyr}$ for Laniakea Supercluster. WUM is a classical model, therefore classical notions can be introduced only when the very first ensemble of particles was created at the cosmological time $\tau_{M} \cong 10^{-18} \mathrm{~s}$. At time $\tau \gg 10^{-18} \mathrm{~s}$ density fluctuations could happen in the Medium of the World filled with DMPs. The heaviest particles DMF1 could collect into a cloud with distances between particles smaller than $R_{W}$. As the result of the weak interaction, clumps of DMF1 will arise. Larger clumps will attract smaller clumps and DMPs and initiate a process of expanding the DM clump followed by growth of surrounding shells made up of other DMPs, up to the maximum mass of the shell made up of DMF4 at the end of Dark Epoch (0.45 Gyr).

The process described above is the formation of the DM Core of Superclusters [12]. DMPs supply not only additional mass $\left(\propto \tau^{3 / 2}\right)$ to Cores, but also additional angular momentum $\left(\propto \tau^{2}\right)$ fueling the overspinning of DM Cores (see Section 4.5). We estimate the number of Supercluster Cores at the end of Dark Epoch to be around $\sim 10^{3}$ [21]. It is unlikely that all of them gave birth to Luminous Superclusters at the same cosmological time being far away from each other.

\subsection{Rotational Fission}

According to WUM, a rotational angular momentum of overspinning object before rotational fission is [12]:

$$
L_{\text {rot }} \propto G^{0.5} M_{M O}^{1.5} R_{M O}^{0.5}
$$

where $M_{M O}$ is a mass of overspinning Macroobject, $R_{M O}$ is its radius. These parameters are time-varying: $G \propto \tau^{-1}, M_{M O} \propto \tau^{3 / 2}$ and $R_{M O} \propto \tau^{1 / 2}$. It follows that the rotational angular momentum of Cores $L_{\text {rot }}$ is proportional to $\tau^{2}$.

Virgo Supercluster (VS) is a mass concentration of galaxies containing Milky Way. At least one hundred galaxy groups and clusters are located within its diameter of 110 million light-years. Considering parameters of DMF4 shell (see Table 2), we calculate the rotational angular momentum $L_{\text {rot }}^{\text {VSC }}$ of VS Core before rotational fission:

$$
L_{\text {rot }}^{V S C}=3.7 \times 10^{77} \mathrm{~J} \cdot \mathrm{s}
$$

Milky Way (MW) is gravitationally bounded with VS [51]. Let us compare $L_{\text {rot }}^{V S C}$ with an orbital momentum of MW $L_{\text {orb }}^{M W}$ calculated based on the distance of sixty-five million light years from VS Core and orbital speed of about 400 $\mathrm{km} / \mathrm{s}[51]$ :

$$
L_{\text {orb }}^{M W}=2.5 \times 10^{71} \mathrm{~J} \cdot \mathrm{s}
$$

It means that as the result of rotational fission of VS Core, approximately $\sim 10^{6}$ 
galaxies like Milky Way could be generated at the same time. Considering that density of galaxies in the VS falls off with the square of the distance from its center and the location of MW on the outskirts of the VS [52], the actual number of created galaxies could be much larger.

Analogous calculations for MW Core based on parameters of DMF3 shell (see Table 2) produce the following value of rotational angular momentum $L_{\text {rot }}^{M W C}$ [12]:

$$
L_{\text {rot }}^{M W C}=2.4 \times 10^{60} \mathrm{~J} \cdot \mathrm{s}
$$

which far exceeds the orbital momentum of the Solar System $L_{\text {orb }}^{\text {SS }}$ calculated based on the distance from the galactic center of 26.4 kly and orbital speed of about $220 \mathrm{~km} / \mathrm{s}$ :

$$
L_{\text {orb }}^{S S}=1.1 \times 10^{56} \mathrm{~J} \cdot \mathrm{S}
$$

As the result of rotational fission of MW Core 13.77 Gyr ago, approximately $\sim 10^{4}$ Extrasolar systems like Solar System could be created at the same time. Considering that MW has grown inside out (in the present Epoch, most old stars can be found in the middle, more recently formed ones on the outskirts [50]), the number of generated Extrasolar systems could be much larger. Extrasolar system Cores can give birth to planetary cores, which in turn can generate cores of moons by the same Rotational Fission mechanism.

The oldest known star HD 140283 (Methuselah star) is a subgiant star about 190 light years away from Earth for which a reliable age has been determined [53]. H. E. Bond, et al. found its age to be $14.46 \pm 0.8 \mathrm{Gyr}$ that does not conflict with the age of the Universe, $13.77 \pm 0.06 \mathrm{Gyr}$, based on the microwave background and Hubble constant [53]. It means that this star must have formed between 13.66 and $13.83 \mathrm{Gyr}$, amount of time that is too short for formation of second generation of stars according to prevailing theories. In our Model, this discovery can be explained by generation of HD 140283 by overspinning Core of the MW 13.77 Gyr ago.

In frames of the developed Rotational Fission model, it is easy to explain hyper-runaway stars unbound from MW with speeds of up to $\sim 700 \mathrm{~km} / \mathrm{s}$ [54]: they were launched by overspinning Core of the Large Magellan Cloud with the speed higher than the escape velocity [12].

\subsection{Luminous Epoch}

Luminous Epoch spans from $0.45 \mathrm{Gyr}$ up to the present Epoch (during 13.77 Gyr). According to WUM, Cores of all Macroobjects (MOs) of the World (Superclusters, Galaxies, Extrasolar systems) possess the following properties [13]:

- Their Nuclei are made up of DMFs and contain other particles, including Dark Matter and Baryonic matter, in shells surrounding the Nuclei;

- DMPs are continuously absorbed by Cores of all MOs. Luminous Matter (about $7.2 \%$ of the total Matter in the World) is a byproduct of DMPs selfannihilation. Luminous Matter is re-emitted by Cores of MOs continuously; 
- Nuclei and shells are growing in time: size $\propto \tau^{1 / 2}$; mass $\propto \tau^{3 / 2}$; and rotational angular momentum $\propto \tau^{2}$, until they reach the critical point of their stability, at which they detonate. Satellite cores and their orbital $L_{\text {orb }}$ and rotational $L_{\text {rot }}$ angular momenta released during detonation are produced by Overspinning Core (OC). The detonation process does not destroy OC; it is rather gravitational hyper-flares;

- Size, mass, composition, $L_{\text {orb }}$ and $L_{\text {rot }}$ of satellite cores depend on local density fluctuations at the edge of OC and cohesion of the outer shell. Consequently, the diversity of satellite cores has a clear explanation.

WUM refers to OC detonation process as Gravitational Burst (GB), analogous

to Gamma Ray Burst [7]. In frames of WUM, the repeating GBs can be explained the following way:

- As the result of GB, the OC loses a small fraction of its mass and a large part of its rotational angular momentum;

- After GB, the Core absorbs new DMPs. Its mass increases $\propto \tau^{3 / 2}$, and its angular momentum $L_{\text {rot }}$ increases much faster $\propto \tau^{2}$, until it detonates again at the next critical point of its stability;

- Afterglow of GBs is a result of processes developing in the Nuclei and shells after detonation;

- In case of Extrasolar systems, a star wind is the afterglow of star detonation: star Core absorbs new DMPs, increases its mass $\propto \tau^{3 / 2}$ and gets rid of extra $L_{\text {rot }}$ by star wind particles;

- Solar wind is the afterglow of Solar Core detonation 4.57 Gyr ago. It creates the bubble of the heliosphere continuously;

- In case of Galaxies, a galactic wind is the afterglow of repeating galactic Core detonations. In Milky Way it continuously creates two Dark Matter Fermi Bubbles (see Section 4.7).

S. E. Koposov, et al. present the discovery of the fastest Main Sequence hyper-velocity star S5-HVS1 with mass about 2.3 solar masses that is located at a distance of $\sim 9 \mathrm{kpc}$ from the Sun. When integrated backwards in time, the orbit of the star points unambiguously to the Galactic Centre, implying that S5-HVS1 was kicked away from Sgr A* with a velocity of $\sim 1800 \mathrm{~km} / \mathrm{s}$ and travelled for 4.8 Myr to the current location. So far, this is the only hyper-velocity star confidently associated with the Galactic Centre [55]. In frames of the developed Model this discovery can be explained by Gravitational Burst of the overspinning Core of the Milky Way 4.8 million years ago, which gave birth to S5-HVS1 with the speed higher than the escape velocity of the Core.

C. J. Clarke, et al. observed CI Tau, a young 2-million-year-old star. CI Tau is located about five hundred light years away in a highly-productive stellar 'nursery' region of the galaxy. They discovered that the Extrasolar System contains four gas giant planets that are only two million years old [56], amount of time that is too short for formation of gas giants according to prevailing theories. In frames of the developed Rotational Fission model, this discovery can be ex- 
plained by Gravitational Burst of the overspinning Core of the Milky Way two million years ago, which gave birth to CI Tau system with all planets generated at the same time [12].

To summarize:

- The rotational fission of Macroobject DM Cores is the most probable process that can generate satellite cores with large orbital momenta in a very short time;

- Macrostructures of the World form from the top (superclusters) down to galaxies, extrasolar systems, planets, and moons;

- Gravitational waves can be a product of rotational fission of overspinning DM Macroobject Cores.

\subsection{Dark Matter Fermi Bubbles}

In 2010, the discovery of two Fermi Bubbles (FBs) emitting gamma- and X-rays was announced. FBs extend for about twenty-five kly above and below the center of the galaxy [57]. The outlines of the bubbles are quite sharp, and the Bubbles glow in nearly uniform gamma rays over their colossal surfaces. Gamma-ray spectrum remains unconstrained up to around $1 \mathrm{TeV}$ [58]. Years after the discovery of FBs, their origin and the nature of the gamma-ray emission remain unresolved.

In WUM, Fermi Bubbles are DMPs' clouds containing uniformly distributed Dark Matter Objects (DMOs), in which DMPs self-annihilate and radiate X-rays and gamma rays. FBs made up of DMF3 particles resemble a honeycomb filled with DMF1 and DMF2. Weak interaction between DMF3 particles provides integrity of FBs. Gamma rays up to $1 \mathrm{TeV}$ are the result of the self-annihilation of DMF1 $(1.3 \mathrm{TeV})$ and DMF2 $(9.6 \mathrm{GeV})$ in DMOs, which are macroobjects whose density is sufficient for the self-annihilation of DMPs to occur. On the other hand, DMOs are much smaller than stars in the World, and have a high concentration in FBs to provide nearly uniform gamma ray glow over their colossal surfaces. The Core of MW supplies FBs with new DMPs through the galactic wind, explaining the brightness of FBs remaining constant during the time of observations. In our opinion, FBs are built continuously throughout the lifetime of MW (13.77 Byr) [14].

\subsection{Dark Matter Cores of Macroobjects}

The following facts support the existence of DM Cores of Macroobjects [12]:

- E. Fossat, et al. found that Solar Core rotates $3.8 \pm 0.1$ faster than the surrounding envelope;

- J. Zhang, et al. concluded that the Earth's inner core is rotating faster than its surface by about 0.3 - 0.5 degrees per year;

- T. Guillot, et al. found that a deep interior of Jupiter rotates nearly as a rigid body, with differential rotation decreasing by at least an order of magnitude compared to the atmosphere. 
A fact that Macroobject Cores rotate faster than surrounding envelopes, despite high viscosity of the internal medium, is intriguing. WUM explains this phenomenon through absorption of DMPs by Cores. Dark Matter particles supply not only additional mass ( $\propto \tau^{3 / 2}$ ), but also additional angular momentum $\left(\propto \tau^{2}\right)$. Cores irradiate products of DMPs self-annihilation, which carry away excessive angular momentum. The Solar wind is the result of this mechanism for the Sun. Upper mantle with Crust for the Earth and other planets and moons are produced by the same mechanism. Radiuses of DM cores of the different Macroobjects of SS are presented in Table 3.

\subsection{Sun's Dark Matter Core}

Internal Structure. According to the standard Solar model, the Sun has:

- Core that extends from the center to about $20 \%-25 \%$ of the solar radius, contains $34 \%$ of the Sun's mass. It produces all of Sun's energy;

- Radiative zone from the Core to about $70 \%$ of the solar radius, in which convection does not occur and energy transfer occurs by means of radiation;

- Core and Radiative zone contain practically all Sun's mass [59].

The large power output of the Sun is mainly due to the huge size and density of its Core, with only a fairly small amount of power being generated per cubic meter. Theoretical models of the Sun's interior indicate a maximum power density of approximately $276.5 \mathrm{~W} / \mathrm{m}^{3}$ at the center of the Core [60], which is about the same power density inside a compost pile [61] and closer approximates reptile metabolism than a thermonuclear bomb. In our view, Core and Radiative zone are the parts of the Sun's DM Core.

Evolution of the Sun. By 1950s, stellar astrophysicists had worked out the physical principles governing the structure and evolution of stars [62]. According to these principles, the Sun's luminosity had to change over time, with the young Sun being about 30\% less luminous than today [63] [64] [65] [66]. The long-term evolution of the bolometric solar luminosity $L(\tau)$ as a function of cosmological time $\tau$ can be approximated by a simple linear law: $L(\tau) \propto \tau$ [62].

One of the consequences of WUM holds that all stars were fainter in the past. As their cores absorb new DMPs, size of MO cores $R_{M O}$ and their luminosity $L_{M O}$ are increasing in time: $R_{M O} \propto \tau^{1 / 2}$ and $L_{M O} \propto R_{M O}^{2} \propto \tau$, respectively. Taking the age of the World: $A_{w} \cong 14.2 \mathrm{Byr}$ and the age of SS: $A_{S S} \cong 4.6 \mathrm{Byr}$, it is easy to find that the young Suns' output was $67 \%$ of what it is today. Literature commonly refers to the value of 70\% [62]. This result supports the developed model of the structure and evolution of the Sun [21].

Table 3. The radius of DM cores of the different Macroobjects in the Solar system [19].

\begin{tabular}{ccccccc}
\hline Macroobject & Sun & Saturn & Earth & Mars & Moon & Mimas \\
\hline Radius, $\mathbf{k m}\left(\times 10^{3}\right)$ & 487 & 34.9 & 3.52 & 1.83 & 0.381 & $<0.2$ \\
\hline
\end{tabular}




\subsection{Solar Corona, Geocorona, Planetary Coronas}

Solar Corona is an aura of plasma that surrounds the Sun and extends at least 8 $\times 10^{6} \mathrm{~km}$ into outer space (compare with the Sun's radius $7 \times 10^{5} \mathrm{~km}$ ). Spectroscopy measurements indicate strong ionization and plasma temperature in excess of $10^{6} \mathrm{~K}$ [67]. The corona emits radiation mainly in the X-rays, observable only from space. The plasma is transparent to its own radiation and to solar radiation passing through it, therefore we say that it is optically-thin. The gas, in fact, is very rarefied, and the photon mean free-path by far overcomes all other length-scales, including the typical sizes of the coronal features.

J. T. Schmelz made the following comment on the composition of Solar corona: Along with temperature and density, the elemental abundance is a basic parameter required by astronomers to understand and model any physical system. The abundances of the solar corona are known to differ from those of the solar photosphere [68].

In WUM, Solar corona made up of DMPs resembles a honeycomb filled with plasma. The following experimental results speak in favor of this model [14]:

- The corona emits radiation mainly in X-rays due to the self-annihilation of DMF3 particles;

- The plasma is transparent to its own radiation and to the radiation coming from below;

- The elemental composition of the Solar corona and the Solar photosphere are known to differ;

- During the impulsive stage of Solar flares, radio waves, hard x-rays, and gamma rays with energy above $100 \mathrm{GeV}$ are emitted [69] (one photon had an energy as high as $467.7 \mathrm{GeV}$ [14]). In our view, it is the result of enormous density fluctuations of DMPs in the Solar corona and their self-annihilation.

Coronal Heating problem in solar physics relates to the question of why the temperature of the Solar corona is millions of degrees higher than that of the photosphere. The high temperatures require energy to be carried from the solar interior to the corona by non-thermal processes.

In our opinion, the origin of the Solar corona plasma is not the coronal heating. Plasma particles (electrons, protons, multicharged ions) are so far apart that plasma temperature in the usual sense is not very meaningful. The plasma is the result of self-annihilation of DMF1 (1.3 TeV), DMF2 (9.6 GeV), and DMF3 (3.7 $\mathrm{keV}$ ) particles. The Solar corona made up of DMPs resembles a honeycomb filled with plasma [12].

Geocorona is a luminous part of an outermost region of the Earth's atmosphere [13] that extends to at least $640,000 \mathrm{~km}$ from the Earth [70]. It is seen primarily via Far-Ultra-Violet light from the Sun that is scattered by neutral hydrogen [71]. X-rays (in the range of energies $0.08-10 \mathrm{keV}$ ) from Earth's Geocorona were first detected by Chandra X-ray Observatory [72].

Planetary Coronas. X-rays from Planets and some observed moons (Europa, Io, Io Plasma Torus, Titan) were also observed by Chandra [72]. According to 
NASA:

- The X-rays from Venus and, to some extent, the Earth, are due to the fluorescence of solar X-rays striking the atmosphere;

- Fluorescent X-rays from oxygen atoms in the Martian upper atmosphere are similar to those on Venus. A huge Martian dust storm was in progress when the Chandra observations were made. The intensity of the X-rays did not change during the dust storm;

- Jupiter has an environment capable of producing X-rays in a different manner because of its substantial magnetic field. X-rays are produced when highenergy particles from the Sun get trapped in its magnetic field and accelerated toward the polar regions where they collide with atoms in Jupiter's atmosphere;

- Like Jupiter, Saturn has a strong magnetic field, so it was expected that Saturn would also show a concentration of X-rays toward the poles. However, Chandra's observation revealed instead an increased X-ray brightness in the equatorial region. Furthermore, Saturn's X-ray spectrum was found to be similar to that of X-rays from the Sun.

In WUM, the Geocorona and Planetary Coronas possess features like those of the Solar Corona.

\subsection{Dark Matter Reactors}

Internal Heating. The analysis of Sun's heat for planets in SS yields the effective temperature of Earth of $255 \mathrm{~K}$ [73]. The actual mean surface temperature of Earth is $288 \mathrm{~K}$ [74]. The higher actual temperature of Earth is due to energy generated internally by the planet itself. According to the standard model, the Earth's internal heat is produced mostly through radioactive decay. The major heat-producing isotopes within Earth are K-40, U-238, and Th-232. The mean global heat loss from Earth is 44.2 TW [75]. The Earth's Uranium has been thought to be produced in one or more supernovae over 6 Gyr ago [76].

Radiogenic decay can be estimated from the flux of geoneutrinos that are emitted during radioactive decay. The KamLAND Collaboration combined precise measurements of the geoneutrino flux with existing measurements from the Borexino detector, Italy. They found that decay of U-238 and Th-232 together contribute about $20 \mathrm{TW}$ to the total heat flux from the Earth to space. The neutrinos emitted from the decay of K-40 contribute 4 TW. Based on the observations the KamLAND Collaboration made a conclusion that heat from radioactive decay contributes about half of Earth's total heat flux [77].

Plutonium-244 with half-life of 80 million years is not produced in significant quantities by the nuclear fuel cycle, because it needs very high neutron flux environments. Any Plutonium-244 present in the Earth's crust should have decayed by now. Nevertheless, D. C. Hoffman, et al. in 1971 obtained the first indication of Pu-244 present existence in Nature [78].

In WUM, all chemical products of the Earth including isotopes K-40, U-238, Th-232, and Pu-244, are produced within the Earth as the result of DMF1 
self-annihilation [13]. They arrive in the Crust of Earth due to convection currents in the mantle carrying heat and isotopes from the interior to the planet's surface [79].

Jupiter radiates more heat than it receives from the Sun [80]. Giant planets like Jupiter are hundreds of degrees warmer than current temperature models predict. Until now, the extremely warm temperatures observed in Jupiter's atmosphere (about 970 degrees $\mathrm{C}$ [81]) have been difficult to explain, due to lack of a known heat source [11]. Saturn radiates 2.5 times more energy than it receives from the Sun [82]; Uranus-1.1 times [83]; Neptune-2.6 times [84].

S. Kamata, et al. report that "many icy Solar System bodies possess subsurface oceans. To maintain an ocean, Pluto needs to retain heat inside." Kamata, et al. show that "the presence of a thin layer of gas hydrates at the base of the ice shell can explain both the long-term survival of the ocean and the maintenance of shell thickness contrasts. Gas hydrates act as a thermal insulator, preventing the ocean from completely freezing while keeping the ice shell cold and immobile. The most likely guest gas is methane" [85].

According to WUM, the internal heating of all gravitationally-rounded objects of the Solar system is due to DMPs self-annihilation in their cores made up of DMF1 $(1.3 \mathrm{TeV})$. The amount of energy produced due to this process is sufficiently high to heat up the objects. New DMF1 freely penetrate through the entire objects' envelope, get absorbed into the cores, and continuously support DMF1 self-annihilation. Objects' cores are essentially Dark Matter Reactors fueled by DMF1 [12].

In WUM, Macroobjects' cores are essentially DM Reactors fueled by DMPs. Chemical elements, compositions, radiations are produced by Macroobjects themselves as the result of DMPs self-annihilation. The diversity of all gravitationally-rounded Macroobjects in the Solar system is explained by the differences in their DM cores (mass, size, density, composition). The DM Reactors at their cores (including Earth) are very efficient and provide enough energy for the internal heating and all their geological processes like volcanos, quakes, mountains' formation through tectonic forces or volcanism, tectonic plates' movements, etc. [21].

\section{Conclusions}

Dark Matter is abundant:

- $2.4 \%$ of Luminous Matter is in Superclusters, Galaxies, Stars, Planets, etc.

- $4.8 \%$ of Luminous Matter is in the Medium of the World;

- The remaining $92.8 \%$ is Dark Matter.

Dark Matter is omnipresent:

- Cores of all Macroobjects;

- Coronas of all Macroobjects of the World;

- The Medium of the World;

- Fermi Bubbles. 
WUM is based on two dimensionless parameters only: Rydberg constant $\alpha$ and time-varying quantity $Q$. In WUM we often use well-known physical parameters, keeping in mind that all of them can be expressed through the Basic Units of time $t_{0}$, size $a$, and energy $E_{0}$. Taking the relative values of physical parameters in terms of the Basic Units we can express all dimensionless parameters of the World through two parameters $\alpha$ and $Q$ in various rational exponents, as well as small integer numbers and $\pi$. There are no Fundamental Physical Constants in WUM. In our opinion, constant $\alpha$ and quantity $Q$ should be named "Universe Constant" and "World Parameter" respectively.

Based on the totality of results obtained by WUM, we suggest adopting the existence of the multicomponent Dark Matter in the World from the Classical Physics point of view. While WUM needs significant further elaboration, it can already serve as a basis for a New Physics proposed by Paul Dirac in 1937.

\section{Acknowledgements}

I am always grateful to Academician Alexander Prokhorov and Prof. Alexander Manenkov, whose influence on my scientific life has been decisive. I am eternally grateful to my Scientific Father Paul Dirac who was a genius and foresaw the Future of Physics in a New Cosmology. I am forever grateful to Nicola Tesla who was a genius. I am much obliged to Prof. Christian Corda for publishing my manuscripts in JHEPGC. Special thanks to my son Ilya Netchitailo who helped me refine the Model and improve its understanding.

\section{Conflicts of Interest}

The author declares no conflicts of interest regarding the publication of this paper.

\section{References}

[1] Netchitailo, V.S. (2013) Word-Universe Model. viXra:1303.0077v7. https://vixra.org/abs/1303.0077

[2] Netchitailo, V.S. (2015) 5D World-Universe Model Space-Time-Energy. Journal of High Energy Physics, Gravitation and Cosmology, 1, 25-34. https://doi.org/10.4236/jhepgc.2015.11003

[3] Netchitailo, V.S. (2015) 5D World-Universe Model. Multicomponent Dark Matter. Journal of High Energy Physics, Gravitation and Cosmology, 1, 55-71. https://doi.org/10.4236/jhepgc.2015.12006

[4] Netchitailo, V.S. (2016) 5D World-Universe Model. Neutrinos. The World. Journal of High Energy Physics, Gravitation and Cosmology, 2, 1-18. https://doi.org/10.4236/jhepgc.2016.21001

[5] Netchitailo, V.S. (2016) 5D World-Universe Model. Gravitation. Journal of High Energy Physics, Gravitation and Cosmology, 2, 328-343. https://doi.org/10.4236/jhepgc.2016.23031

[6] Netchitailo, V.S. (2016) Overview of Hypersphere World-Universe Model. Journal of High Energy Physics, Gravitation and Cosmology, 2, 593-632. https://doi.org/10.4236/jhepgc.2016.24052 
[7] Netchitailo, V.S. (2017) Burst Astrophysics. Journal of High Energy Physics, Gravitation and Cosmology, 3, 157-166. https://doi.org/10.4236/jhepgc.2017.32016

[8] Netchitailo, V.S. (2017) Mathematical Overview of Hypersphere World-Universe Model. Journal of High Energy Physics, Gravitation and Cosmology, 3, 415-437. https://doi.org/10.4236/jhepgc.2017.33033

[9] Netchitailo, V.S. (2017) Astrophysics: Macroobject Shell Model. Journal of High Energy Physics, Gravitation and Cosmology, 3, 776-790. https://doi.org/10.4236/jhepgc.2017.34057

[10] Netchitailo, V.S. (2018) Analysis of Maxwell's Equations. Cosmic Magnetism. Journal of High Energy Physics, Gravitation and Cosmology, 4, 1-7. https://doi.org/10.4236/jhepgc.2018.41001

[11] Netchitailo, V.S. (2018) Hypersphere World-Universe Model. Tribute to Classical Physics. Journal of High Energy Physics, Gravitation and Cosmology, 4, 441-470. https://doi.org/10.4236/jhepgc.2018.43024

[12] Netchitailo, V.S. (2019) Solar System. Angular Momentum. New Physics. Journal of High Energy Physics, Gravitation and Cosmology, 5, 112-139.

https://doi.org/10.4236/jhepgc.2019.51005

[13] Netchitailo, V.S. (2019) High-Energy Atmospheric Physics: Ball Lightning. Journal of High Energy Physics, Gravitation and Cosmology, 5, 360-374. https://doi.org/10.4236/jhepgc.2019.52020

[14] Netchitailo, V.S. (2019) Dark Matter Cosmology and Astrophysics. Journal of High Energy Physics, Gravitation and Cosmology, 5, 999-1050. https://doi.org/10.4236/jhepgc.2019.54056

[15] Netchitailo, V.S. (2020) World-Universe Model-Alternative to Big Bang Model. Journal of High Energy Physics, Gravitation and Cosmology, 6, 133-258. https://doi.org/10.4236/jhepgc.2020.61012

[16] Netchitailo, V.S. (2020) World-Universe Model Predictions. Journal of High Energy Physics, Gravitation and Cosmology, 6, 282-297. https://doi.org/10.4236/jhepgc.2020.62022

[17] Netchitailo, V.S. (2020) Hypersphere World-Universe Model: Basic Ideas. Journal of High Energy Physics, Gravitation and Cosmology, 6, 710-752. https://doi.org/10.4236/jhepgc.2020.64049

[18] Netchitailo, V.S. (2021) Hypersphere World-Universe Model: Evolution of the World. Journal of High Energy Physics, Gravitation and Cosmology, 7, 508-530. https://doi.org/10.4236/jhepgc.2021.72029

[19] Netchitailo, V.S. (2021) Hypersphere World-Universe Model. Journal of High Energy Physics, Gravitation and Cosmology, 7, 915-941. https://doi.org/10.4236/jhepgc.2021.72042

[20] Netchitailo, V.S. (2021) Solar System. Angular Momentum. Dark Matter Reactors. Journal of High Energy Physics, Gravitation and Cosmology, 7, 1353-1372. https://doi.org/10.4236/jhepgc.2021.74084

[21] Netchitailo, V.S. (2021) From the Beginning of the World to the Beginning of Life on Earth. Journal of High Energy Physics, Gravitation and Cosmology, 7, 1503-1523. https://doi.org/10.4236/jhepgc.2021.74092

[22] Bertone, G. and Hooper, D. (1916) A History of Dark Matter. arXiv:1605.04909.

[23] Orodruin (2016) Learn about Knut Lundmark and the Prehistory of Dark Matter. Physics Forums Insights.

https://www.physicsforums.com/insights/knut-lundmark-prehistory-dark-matter/ 
[24] Lee, B.W. and Weinberg, S. (1977) Cosmological Lower Bound on Heavy-Neutrino Masses. Physical Review Letters, 39, 165-168. https://journals.aps.org/prl/abstract/10.1103/PhysRevLett.39.165 https://doi.org/10.1103/PhysRevLett.39.165

[25] Dicus, D.A., Kolb, E.W. and Teplitz, V.L. (1977) Cosmological Upper Bound on Heavy-Neutrino Lifetimes. Physical Review Letters, 39, 168-171.

https://doi.org/10.1103/PhysRevLett.39.168

[26] Dicus, D.A., Kolb, E.W. and Teplitz, V.L. (1978) Cosmological Implications of Massive, Unstable Neutrinos. The Astrophysical Journal, 221, 327-341. https://doi.org/10.1086/156031

[27] Gunn, J.E., et al. (1978) Some Astrophysical Consequences of the Existence of a Heavy Stable Neutral Lepton. The Astrophysical Journal, 223, 1015-1031. https://doi.org/10.1086/156335

[28] Stecker, F.W. (1978) The Cosmic Gamma-Ray Background from the Annihilation of Primordial Stable Neutral Heavy Leptons. The Astrophysical Journal, 223, 1032 1036. https://doi.org/10.1086/156336

[29] Zeldovich, Y.B., Klypin, A.A., Khlopov, M.Y. and Chechetkin, V.M. (1980) Astrophysical Constraints on the Mass of Heavy Stable Neutral Leptons. Soviet Journal of Nuclear Physics, 31, 664-669.

[30] Ripamonti, E. and Abel, T. (2005) The Formation of Primordial Luminous Objects. arXiv:0507130.

[31] Spolyar, D., Freese, K. and Gondolo, P. (2007) Dark Matter and the First Stars: A New Phase of Stellar Evolution. Physical Review Letters, 100, Article ID: 051101.

[32] Freese, K., Rindler-Daller, T., Spolyar, D. and Valluri, M. (2015) Dark Stars: A Review. Reports on Progress in Physics, 79, Article ID: 066902.

https://doi.org/10.1088/0034-4885/79/6/066902

[33] Corda, C. (2009) Interferometric Detection of Gravitational Waves: The Definitive Test for General Relativity. International Journal of Modern Physics D, 18, 22752282. https://doi.org/10.1142/S0218271809015904

[34] Boehm, C., Fayet, P. and Silk, J. (2003) Light and Heavy Dark Matter Particles. Physical Review D, 69, Article ID: 101302. https://doi.org/10.1103/PhysRevD.69.101302

[35] Aoki, M., et al. (2012) Multicomponent Dark Matter Systems and Their Observation Prospects. Physical Review D, 86, Article ID: 076015.

[36] Zurek, K.M. (2009) Multicomponent Dark Matter. Physical Review D, 79, Article ID: 115002. https://doi.org/10.1103/PhysRevD.79.115002

[37] Feng, J.L. (2010) Dark Matter Candidates from Particle Physics and Methods of Detection. Annual Review of Astronomy and Astrophysics, 48, 495-545. https://doi.org/10.1146/annurev-astro-082708-101659

[38] Feldman, D., Liu, Z., Nath, P. and Peim, G. (2010) Multicomponent Dark Matter in Supersymmetric Hidden Sector Extensions. Physical Review D, 81, Article ID: 095017. https://doi.org/10.1103/PhysRevD.81.095017

[39] Heeck, J. and Zhang, H. (2012) Exotic Charges, Multicomponent Dark Matter and Light Sterile Neutrinos. Journal of High Energy Physics, 2013, Article No. 164. https://doi.org/10.1007/JHEP05(2013)164

[40] Feng, W.Z., Mazumdar, A. and Nath, P. (2013) Baryogenesis from Dark Matter. Physical Review D, 88, Article ID: 036014. https://doi.org/10.1103/PhysRevD.88.036014

[41] Bliss, L. (2014) The Milky Way's ‘City' Just Got a New Name. Bloomberg CityLab. 
https://www.bloomberg.com/news/articles/2014-09-03/the-milky-way-s-city-just-go t-a-new-name

[42] Heymans, C., et al. (2008) The Dark Matter Environment of the Abell 901/902 Supercluster: A Weak Lensing Analysis of the HST STAGES Survey. Monthly Notices of the Royal Astronomical Society, 385, 1431-1442. https://doi.org/10.1111/j.1365-2966.2008.12919.x

[43] Zwicky, F. (1933) Die Rotverschiebung von extragalaktischen Nebeln. Helvetica Physica Acta, 6, 110-127.

[44] Tully, R.B., et al. (2014) The Laniakea Supercluster of Galaxies. Nature, 513, 71-73. https://doi.org/10.1038/nature13674

[45] Wang, P., et al. (2021) Possible Observational Evidence that Cosmic Filaments Spin. Nature Astronomy, 5, 839-845. https://doi.org/10.1038/s41550-021-01380-6

[46] Boardman, L. (2021) Discovery of a Giant Arc in Distant Space Adds to Challenges to Basic Assumptions about the Universe. University of Central Lancashire (UCLan), Preston. https://www.star.uclan.ac.uk/ alopez/aas238 press release.pdf

[47] Carr, B., Kühnel, F. and Visinelli, L. (2021) Constraints on Stupendously Large Black Holes. Monthly Notices of the Royal Astronomical Society, 501, 2029-2043. https://doi.org/10.1093/mnras/staa3651

[48] Dirac, P.A.M. (1974) Cosmological Models and the Large Numbers Hypothesis. Proceedings of the Royal Society of London. Series A, 338, 439-446.

[49] Riemann, B. (1854) On the Hypotheses Which Lie at the Bases of Geometry. Nature, 8, 14-17, 36, 37.

[50] Ness, M., et al. (2015) The Cannon: A Data-Driven Approach to Stellar Label Determination. The Astrophysical Journal, 808, 16.

https://doi.org/10.1088/0004-637X/808/1/16

[51] NASA (2015) The Cosmic Distance Scale. https://imagine.gsfc.nasa.gov/features/cosmic/local supercluster info.html

[52] Tully, R.B. (1982) The Local Supercluster. The Astrophysical Journal, 257, 389-422. https://doi.org/10.1086/159999

[53] Bond, H.E., et al. (2013) HD 140283: A Star in the Solar Neighborhood that Formed Shortly after the Big Bang. The Astrophysical Journal Letters, 765, L12. https://doi.org/10.1088/2041-8205/765/1/L12

[54] Marchetti, T., Rossi, E.M. and Brown. A.G.A. (2018) Gaia DR2 in 6D: Searching for the Fastest Stars in the Galaxy. Monthly Notices of the Royal Astronomical Society, 490, 157-171. https://doi.org/10.1093/mnras/sty2592

[55] Koposov, S.E., et al. (2019) The Great Escape: Discovery of a Nearby $1700 \mathrm{~km} / \mathrm{s} \mathrm{Star}$ Ejected from the Milky Way by Sgr A*. Monthly Notices of the Royal Astronomical Society, 491, 2465-2480. https://doi.org/10.1093/mnras/stz3081

[56] Clarke, C.J., et al. (2018) High-Resolution Millimeter Imaging of the CI Tau Protoplanetary Disk: A Massive Ensemble of Protoplanets from 0.1 to $100 \mathrm{au}$. The Astrophysical Journal Letters, 866, L6. https://doi.org/10.3847/2041-8213/aae36b

[57] Aguilar, D.A. and Pulliam, C. (2010) Astronomers Find Giant, Previously Unseen Structure in our Galaxy. Harvard-Smithsonian Center for Astrophysics.

[58] Yang, L. and Razzaque, S. (2019) Constraints on Very High Energy Gamma-Ray Emission from the Fermi Bubbles with Future Ground-Based Experiments. Physical Review D, 99, Article ID: 083007. https://doi.org/10.1103/PhysRevD.99.083007

[59] Djorgovski, S.G. (2016) Stellar Structure and the Sun. http://www.astro.caltech.edu/ george/ay1/lec pdf/Ay1 Lec08.pdf 
[60] Cohen, H. (2001) From Core to Corona: Layers of the Sun. Library of Congress Web Archives.

http://webarchive.loc.gov/all/20011129122524/http\%3A//fusedweb.llnl.gov/cpep/ch art pages/5.plasmas/sunlayers.html

[61] Kruszelnicki, K.S. (2012) Lazy Sun Is Less Energetic than Compost. https://www.abc.net.au/science/articles/2012/04/17/3478276.htm

[62] Feulner, G. (2012) The Faint Young Sun Problem. Reviews of Geophysics, 50, 1-30. https://doi.org/10.1029/2011RG000375

[63] Hoyle, F. (1958) Remarks on the Computation of Evolutionary Tracks. Ricerche Astronomiche, 5, 223.

[64] Schwarzschild, M. (1958) Structure and Evolution of the Stars. Princeton University Press, Princeton. https://doi.org/10.1515/9781400879175

[65] Newman, M.J. and Rood, R.T. (1977) Implications of Solar Evolution for the Earth's Early Atmosphere. Science, 198, 1035-1037.

https://doi.org/10.1126/science.198.4321.1035

[66] Fox, K.C. (2014) NASA's STEREO Maps Much Larger Solar Atmosphere than Previously Observed. NASA.

https://www.nasa.gov/content/goddard/nasas-stereo-maps-much-larger-solar-atmo sphere-than-previously-observed/

[67] Aschwanden, M.J. (2004) Physics of the Solar Corona: An Introduction. Praxis Publishing, Singapore.

[68] Schmelz, J.T., et al. (2012) Composition of the Solar Corona, Solar Wind, and Solar Energetic Particles. The Astrophysical Journal, 755, Article No. 33. http://iopscience.iop.org/article/10.1088/0004-637X/755/1/33/pdf https://doi.org/10.1088/0004-637X/755/1/33

[69] Grossman, L. (2018) Strange Gamma Rays from the Sun May Help Decipher Its Magnetic Fields. Science News, 194, No. 6. https://www.sciencenews.org/article/strange-gamma-rays-sun-magnetic-fields

[70] Baliukin, I.I., et al. (2019) SWAN/SOHO Lyman- $\alpha$ Mapping: The Hydrogen Geocorona Extends Well beyond the Moon. Journal of Geophysical Research: Space Physics, 124, 861-885. https://doi.org/10.1029/2018JA026136

[71] Reyes, R. (2012) Exploring the Sun-Earth Connection. Southwest Research Institute. http://pluto.space.swri.edu/image/glossary/geocorona.html

[72] NASA (2012) Solar System. http://chandra.harvard.edu/xray sources/solar system.html

[73] Cole, G.H.A. and Woolfson, M.M. (2002) Planetary Science: The Science of Planets around Stars. Taylor \& Francis, New York. https://doi.org/10.1201/9781420056853

[74] Kinver, M. (2009) Global Average Temperature May Hit Record Level in 2010. BBC.

[75] Pollack, H.N., Hurter, S.J., Johnson, J.R. (1993) Heat Flow from the Earth's Interior: Analysis of the Global Data Set. Reviews of Geophysics, 31, 267-280. https://doi.org/10.1029/93RG01249

[76] Arculus, R. (2016) The Cosmic Origins of Uranium. World Nuclear Association. http://www.world-nuclear.org/information-library/nuclear-fuel-cycle/uranium-reso urces/the-cosmic-origins-of-uranium.aspx

[77] Gando, A., et al. (2011) Partial Radiogenic Heat Model for Earth Revealed by Geoneutrino Measurements. Nature Geoscience, 4, 647-651. https://doi.org/10.1038/ngeo1205 
[78] Hoffman, D.C., et al. (1971) Detection of Plutonium-244 in Nature. Nature, 234, 132-134. https://doi.org/10.1038/234132a0

[79] Ricard, Y. (2009) 7.02-Physics of Mantle Convection. In: Schubert, G., Ed., Treatise on Geophysics, Elsevier, Oxford, UK, 23-71.

[80] Elkins-Tanton, L.T. (2006). Jupiter and Saturn. Chelsea House, New York.

[81] O’Donoghue, J., Moore, L., Stallard, T.S. and Melin, H. (2016) Heating of Jupiter's Upper Atmosphere above the Great Red Spot. Nature, 536, 190-192. https://doi.org/10.1038/nature18940

[82] de Pater, I. and Lissauer, J.J. (2010) Planetary Sciences. 2nd Edition, Cambridge University Press, Cambridge, 254-255.

[83] Class 12-Giant Planets-Heat and Formation. 3750-Planets, Moons \& Rings. Colorado University, Boulder.

[84] Pearl, J.C. and Conrath, B.J. (1991). The Albedo, Effective Temperature, and Energy Balance of Neptune, as Determined from Voyager Data. Journal of Geophysical Research: Space Physics, 96, 18921-18930. https://doi.org/10.1029/91JA01087

[85] Kamata, S., et al. (2019) Pluto's Ocean Is Capped and Insulated by Gas Hydrates. Nature Geoscience, 12, 407-410. https://doi.org/10.1038/s41561-019-0369-8 